\title{
Fibrinogen Decreased, CTCAE
}

National Cancer Institute

\section{Source}

National Cancer Institute. Fibrinogen Decreased, CT CAE. NCI Thesaurus. Code C143486.

A finding based on laboratory test results that indicate an decrease in levels of fibrinogen

in a blood specimen. 